Warlow performed a case-control comparison of 114 patients with transient global amnesia, 109 normal community based controls, and 212 patients with transient ischaemic attacks. ${ }^{2}$ The results of this careful study do not support a cerebrovascular cause for transient global amnesia. Firstly, there were no significant differences in the prevalence of vascular risk factors between the patients with transient global amnesia and normal controls; secondly, those with transient global amnesia and those with transient ischaemic attack were not matched for vascular risk factors; and, thirdly, during follow up patients subject to transient ischaemic attacks had a much greater incidence of vascular events and a higher mortality than those subject to transient global amnesia. On the other hand, migraine was more common in those with transient global amnesia than in those in both control groups, though there were no differences in the prevalence of epilepsy.

Unfortunately this information does not enable us to reach a firm conclusion about the cause of transient global amnesia, which in most cases remains uncertain. Thromboembolic disease may be the cause in a few cases, and migraine and epilepsy might account for some more. At a practical level no treatment will reduce the risk of recurrence, though the condition is benign and in general the patient can be reassured. Up to now, believing the transient ischaemic attack theory, my own advice to patients has been that they should not drive for six months after an episode of amnesia. Based on the recent evidence, however, the licensing authority in Swansea now recommends no restrictions to holders of an ordinary driving licence and a one year restriction to holders of a vocational licence.

Senior Lecturer in Neurology,

N E F CARTLIDGE

School of Neurosciences,

University of Newcastle upon Tyne,

Newcastle upon Tyne NE2 4HH

1 Fisher CM, Adams RD. Transient global amnesia. Acta Neurol Scand 1964;40(suppl 9):1-83.

2 Hodges JR, Warlow CP. The aetiology of transient global amnesia. A case-control study of 114 cases with prospective follow up. Brain 1990;113:639-57.

3 Hodges JR, Ward CD. Observations during transient global amnesia. Brain 1989;112:595-620.

4 Kaplan LB. Transient global amnesia. In: Vinken PJ, Bruyn GW, Klawans HL, eds. Handbook of clinical neurology. vol 45. Amsterdam: Elsevier, 1985:205-18.

Fisher CM. Transient global amnesia; precipitating activities and other observations. Arch Neurol 1982;39:605-8.

6 Kaplan L, Chedru F, L'Hermitte F, Mayman C. Transient global amnesia and migraine. Neurology 1981;31:1169-70.

\title{
Surrogate end points in clinical trials
}

\author{
Getting closer to identifying markers for survival in AIDS
}

The ultimate goals of developing new treatments for a fatal disease are, in order of priority: cure, prolonging life, and reducing morbidity without adversely affecting survival. The data required to assess these goals are often obtainable only after a long period of patient follow up as we must observe a certain number of deaths to estimate the survival time reliably.

Unfortunately, the study of fatal diseases generates the greatest pressure to evaluate new drugs rapidly. The desire to reach conclusions about drugs without waiting for survival differences to be definitively established naturally leads to a search for other, more proximal events that might equally well (or even better) reflect therapeutic benefit. Examples of such surrogate end points in common use are blood pressure (for cardiovascular mortality) ${ }^{1}$ and tumour enlargement and relapse (for cancer mortality). ${ }^{2}$ Surrogate end points are also used in non-fatal chronic diseases of organ systems - for example, intraocular pressure is used as a surrogate for long term visual function. ${ }^{3}$ These end points are generally accepted as valid for assessing therapeutic efficacy and have served as the basis for regulatory agencies' approval of drugs.

Clinical trials in AIDS are already using surrogate end points. Recently completed trials of zidovudine in patients in the early stages of HIV infection used progression of disease rather than death as the primary end point. ${ }^{+5}$ In a continuing placebo controlled trial of zidovudine in asymptomatic patients with adequate numbers of CD4 + cells a decline to a count of $0.5 \times 10^{9} / 1$ or below has been defined as a primary end point because zidovudine has already been shown to be effective for patients at these lower counts. Antiviral drug trials in patients with more advanced disease, however, have generally remained dependent on mortality as the primary end point.

Certain biological markers have aroused intense interest as surrogates for survival time in evaluating new treatments for AIDS and HIV infection. The effect of a drug on these markers tends to be observable quickly, often within a few weeks after starting treatment. Several such markers have been shown to be strongly associated with the probability of long term survival, with the greatest attention given to the CD4+ count. ${ }^{6}$ Nevertheless, a correlation between a marker value and ultimate survival time does not necessarily imply that a treatment's effect on the marker will predict the treatment's effect on survival. ${ }^{7}$ For example, a treatment might stabilise or even improve the marker value while producing other adverse effects that tend to shorten survival. A recent example of just such a finding outside AIDS is instructive. Arrhythmias occurring after myocardial infarction are known to be associated with poorer survival; yet a clinical trial of effective antiarrhythmic drugs showed that patients who took these drugs were more likely to die than those who received placebo. ${ }^{8}$ Another concern is that effects on markers may be insufficiently sustained to produce a measurable improvement in clinical outcome.

How, then, can we assess the validity of a potential surrogate marker for trials of AIDS treatment? Certainly there should be a strong biological rationale, and it should also be evident that the marker value at a given point in time is strongly predictive of ultimate survival time. Both of these criteria are met by several markers. What is also required, and what has not been shown for any marker, is that the effect of treatment on the surrogate end point will reliably predict the effect of treatment on survival for individual patients. The paper by Jacobson et al (p 73) takes an important step in this direction. ${ }^{9}$ The authors have gone beyond simply showing the prognostic values of the markers and have investigated whether a change in marker values over time correlates with long term survival. In their analysis of 90 patients receiving zidovudine during trials only changes in $\beta_{2}$ microglobulin concentrations were associated with survival time after they had adjusted for selected baseline characteristics. Absolute CD4+ counts by weeks 8-12-but not changes from baseline counts-provided additional predictive value. p24 antigen concentrations, whether considered as changes from baseline 
or as absolute values at 12 weeks, showed a surprising lack of correlation with long term survival.

This study does, however, have some important limitations. The most obvious is that the sample size is too small to support reliable conclusions about the relative importance of the different markers considered, particularly given the variability of marker measurements. A second limitation is that all patients were treated with zidovudine, preventing direct assessment of the degree to which marker changes were therapeutically induced. Thirdly, these results may not apply at all to drugs that have different mechanisms of action. None the less, these data do begin to suggest that for zidovudine or similar drugs biological markers may be useful in establishing therapeutic efficacy.

Additional investigations of this sort are urgently needed. Particularly important will be analyses on the ever growing bodies of data from completed clinical trials, especially those trials with placebo controls. Careful examination of larger data sets will be needed to confirm the general principle suggested here-that marker values can serve as meaningful end points in drug trials - and to clarify the roles of the various markers that have been studied.

SUSAN S ELLENBERG

Chief, Biostatistics Research Branch,

Division of AIDS, National Institute of Allergy and Infectious Diseases,

Bethesda, Maryland 20892, United States

1 Wittes J, Lakatos E, Probstfield J. Surrogate endpoints in clinical trials: cardiovascular diseases. Stat Med 1989;8:415-26.

2 Ellenberg SS, Hamilton JM. Surrogate endpoints in clinical trials: cancer. Stat Med 1989;8:405-14

3 Hillis A, Seigel D. Surrogate endpoints in clinical trials: ophthalmologic disorders. Stat Med $1989 ; 8: 427-30$.

4 Volberding PA, Lagakos SW, Koch MA, et al. Zidovudine in asymptomatic human immunodeficiency virus infection. N Engl f Med 1990;322:941-9.

5 Fischl MA, Richman DD, Hansen N, et al. The safety and efficacy of zidovudine (AZT) in the treatment of subjects with mildly symptomatic human immunodeficiency virus type 1 (HIV) infection. Ann Intern .Med 1990;112:727-37.

6 Weiss R, Mazade L, eds. Surrogate endpoints in evaluating the effectiveness of drugs against HIV infection and AIDS. Washington, DC: National Academy Press, 1990. (Institute of Medicine, September $11-121989$ conference summary $)$

7 Machado SG, Gail MH, Ellenberg SS. On the use of laboratory markers as surrogates for clinical endpoints in the evaluation of treatment for HIV infection. $\mathcal{F}$ Acquir Immune Defic Syndr 1990;3:1065-73.

8 The Cardiac Arrhythmia Suppression Trial (CAST) Investigators. Preliminary report: effect of encainide and flecainide on mortality in a randomized trial of arrhythmia suppression after myocardial infarction. N Engl f Med 1989;321:406-12.

9 Jacobson MA, Bacchetti P, Kolokathis A, et al. Surrogate markers for survival in patients with AIDS and AIDS related complex treated with zidovudine. BMF 1991;302:73-8.

\section{"Taken from this place and hanged by the neck..."}

\section{Reduction in prison suicides demands cultural change}

Just before Christmas the British parliament once again voted not to reintroduce the death penalty. But while this debate endlessly recurs, more and more prisoners are taking their own lives - almost always by hanging. ${ }^{1}$ It was in 1986 that the Home Office produced its last report on reducing suicides in prison, ${ }^{2}$ and it was in 1987 that suicides more than doubled (from 21 to 46 ). ${ }^{3}$ Clearly the strategy suggested was a failure because suicides have continued to run at roughly twice the number of the early '80s: 48 prisoners killed themselves in prisons in England and Wales in 1990. One response to the problem has been a second report on suicides from the chief inspector of prisons, ${ }^{3}$ and this one contains the seeds of a solution.

Stephen Tumim, a judge, is the second chief inspector, and he has had enormous impact in his short term. With his bow ties, love of art, and inability to drive he has something of the air of a well bred innocent. This manner fools many and may have fooled the Home Office apparatchiks into appointing a chief inspector whom they thought would be unlikely to cause trouble. But Tumim's blunt, well argued, detailed, and highly readable reports have begun to shake the Home Office out of its complacency. Margaret Thatcher is said to have read his report on integral sanitation (prisonspeak for toilets in cells) and been convinced by its argument when the Home Office was wavering. And the new Home Secretary, Kenneth Baker, is reported to have torn up his department's inadequate response to Tumim's latest report and made his bureaucrats start again. ${ }^{+}$The fact that the Home Office put out a new guide on suicide prevention a week before the publication of the chief inspector's report shows how nervous it was.

Suicide in prison is not a new problem. A Parkhurst doctor observed in 1913 that the suicide rate of prisoners was three times that of the general population, ${ }^{5}$ and Topp showed that suicides were commoner among prisoners in $1880(60 / 100000)$ than in $1970(40 / 100000) .{ }^{6}$ A 1990 review confirms, however, a steady increase from the early ' 70 s, and the rate is now higher $(90 / 100000)$ than in $1880 .^{1}$ Comparisons with rates in the general population are obviously fraught with difficulty, and high rates of suicide might be expected among socially disadvantaged young men, many of whom have mental health and drug problems, at a time when they have been convicted of a crime and had their liberty taken away.

The Home Office tends towards a complacent response with, for instance, its assertion after the report on suicides at Risley that "No prison establishment can guarantee that suicide will not occur. The process of incarceration and the mental state of a significant proportion of the inmate population makes such a guarantee impossible." The Home Secretary makes a similar point in the first paragraph of his response to the latest report, ${ }^{8}$ and later he points out that suicides among men under 25 outside custody increased by $20 \%$ between 1987 and 1988 . There will inevitably be some suicides in prison, but all this smacks of defensiveness.

The chief inspector notes this defensiveness when he comments on the prison department's guidelines on suicide prevention, which are primarily concerned with identifying those at risk of suicide and stopping them. The department's circulars are more of a bureaucratic than a human response to the problem. "There was," says the chief inspector, "an overwhelming suspicion that [the procedures] were too defensive. The Circular Instruction was felt to be much more effective at setting out formal procedures rather than in encouraging proper attitudes to inmates." The inadequacy of this response is made complete by the fact that "only a few establishments are able to fulfil both the spirit and the letter of the Circular Instruction." Resources are inadequate, and staff are too poorly trained.

Trying to screen out suicidal prisoners is in itself not enough. The literature review of suicide and self injury published by the Home Office last year points out that most researchers are sceptical about being able to predict suicides. ${ }^{9}$ It isn't easy even in the best conditions. An American psychiatrist concluded after a prospective study of 4800 patients in psychiatric hospitals that "Identification of particular persons who will commit suicide is not currently feasible, because of the low sensitivity and specificity of 